States. Studies of underground miners, who often were exposed to high concentrations of radon, have been used to estimate public health impacts of domestic radon exposure. The healthy worker survivor bias - a condition resulting when individuals in relatively good health tend to work longer and thus become more exposed than individuals in relatively poor health - may be influencing estimates of occupational radon impacts on lung cancer, but this bias has not been thoroughly explored.

Method We implement G-estimation of a structural nested accelerated failure time model to adjust for time-varying confounding by employment history to partially control the healthy worker survivor bias in the Colorado Plateau uranium miners cohort.

Results 615 miners in our cohort died of lung cancer. Assuming no time-varying confounding, we estimate a time ratio $(95 \%$ confidence intervals) per 1000 working level months of exposure of $1.55(1.53,1.58)$, and $1.95(1.86,2.04)$ when to control healthy worker survivor bias, a relative increase of $126 \%$. Estimates of the radon-associated excess cases were 118 under standard methods and 179 when we controlled for the healthy worker survivor bias.

Conclusions There is evidence of a healthy worker survivor bias in standard analyses of the radon-lung cancer association in this cohort. The findings suggest need for further consideration of current estimates of the health impact of radon in occupational and environmental settings.

\section{THE NIEHS GULF STUDY: MENTAL HEALTH SYMPTOMS AMONG PARTICIPANTS INVOLVED IN THE DEEPWATER HORIZON OIL SPILL CLEAN-UP}

${ }^{1}$ Richard Kwok, 1,2Lawrence Engel, 'Christine Ekenga, ${ }^{1}$ Aubrey Miller, ${ }^{3}$ Aaron Blair, 'Dale Sandler. "National Institute of Environmental Health Sciences, Research Triangle Park, NC, USA; ' University of North Carolina at Chapel Hill, Chapel Hill, NC, USA; ${ }^{3}$ National Cancer Institute, Research Triangle Park, NC, USA

\subsection{6/oemed-2014-102362.90}

Objectives Workers and communities impacted by previous oil spills have shown increases in adverse mental health outcomes. The GuLF STUDY is investigating potential health effects among workers involved in the Deepwater Horizon oil spill clean-up response. Participants confronted physical and psychosocial stressors including exposures to oil and dispersants, income uncertainties, and challenges of family and community disruption.

Method Information on demographics, health, and clean-up experience was collected by telephone. Standardised surveys administered to 11210 participants during home visits captured mental health outcomes including depression, anxiety, PTSD, resiliency, and perceived stress. A summary measure of adverse mental health was defined as having a poor outcome on at least one of the five standardised scales. Mental health outcomes were evaluated in relation to clean-up jobs in models that excluded individuals with pre-existing doctor-diagnosed mental health conditions and controlled for socioeconomic and other factors that contribute to mental well-being.

Results Preliminary analysis using the summary mental health measure indicate that persons who worked on oil-spill cleanup were more likely to report adverse mental health outcomes than those who did not, with ORs of 1.4 (95\% CI: 1.1-1.9) for rig and barge workers who worked closer to the source of the oil spill and 1.3 (95\% CI: 1.1-1.5) for those with land-based clean-up jobs compared to those who did not actively work on the clean-up effort.
Conclusions Adverse mental health outcomes were found among individuals in the GuLF STUDY population but further work is necessary to clarify the factors leading to these outcomes.

\section{PRESSURE PAIN SENSITIVITY AND STRESS}

${ }^{1}$ Milan Tucek, ${ }^{2}$ Soren Ballegaard. ${ }^{1}$ First Faculty of Medicine, Institute of Hygiene and Epidemiology, Charles University, Prague, Czech Republic; ${ }^{2}$ UII Care, Hellerup, Denmark

\subsection{6/oemed-2014-102362.91}

Objectives During clinical observations of patients with heart diseases and stress related disorders, it has been observed increased pain sensitivity on specific locations on the skin of the sternum.

Method This sensitivity was measured as the pressure pain sensitivity (PPS) by Ull Metre instrument. Measured PPS values 60 or more indicate high PPS, values below 40 indicate low PPS.

Results There are presented results of PPS measurements in 371 men (av. age $43.6+10.4$ years, 19-66 years); 345 of them were without diagnosis of disease. Average PPS values (whole group) were $36.6+9.5$ (1. measurement) and $36.7+8.5$ (2. measurement) $(\mathrm{r}=0.89)$. Road drivers $(177$ men, PPS values $35.7+9.4$, resp. $36.4+10.9)$ were not significantly different against other occupations (194 men, PPS values $36.5+9.5$, resp. $37.8+11.4$ ). Men with neuropsychological disorders were statistically significantly different against asymptomatic men (PPS values $50.8+$ 14.8 , resp. $67.3+11.4$ vs. $38.8+13.3$, resp. $35.5+5.9, \mathrm{p}=$ 0.002 , resp. less than 0.001 ) and also against men with different diagnosis (PPS values $50.8+14.8$, resp. $67.3+11.4$ vs. $38.8+$ 13.3, resp. $43.4+19.7, p=0.015$, resp. 0.001). Men with other than neuropsychological symptoms doesn't differ significantly in PPS values against asymptomatic men.

Conclusions Method of measurement of PPS could be helpful in medical fitness assessment to work in safety related occupations and is useful for health promotion intervention program. Supported by research project of Charles University in Prague PRVOUK P25/LF1/2.

\section{IDENTIFICATION OF SHORT-TERM, LONG-TERM AND LIFELONG DNA METHYLATION MARKERS OF EXPOSURE TO TOBACCO SMOKE: EVIDENCE FROM EPIC AND NOWAC STUDIES}

${ }^{1}$ Florence Guida, ${ }^{1}$ Gianluca Campanella, ${ }^{2}$ Torkjel Sandanger, ${ }^{2}$ Eiliv Lund, ${ }^{3}$ Roel Vermeulen, 'Paolo Vineis, 'Marc Chadeau-Hyam. 'Imperial College London, London, UK; ${ }^{2}$ The Arctic University of Norway, Tromso, Norway; ${ }^{3}$ Utrecht University, Utrecht, The Netherlands

\subsection{6/oemed-2014-102362.92}

Objectives The aim of our study is to validate and complement recently reported epigenetic biomarkers of exposure to tobacco smoke based on data from two cohorts and to characterise their prospective nature.

Method We used case-control data from studies nested in two prospective cohorts: the Italian component of the European Prospective Investigation into Cancer and Nutrition study $(\mathrm{N}=620)$ and the Norwegian Women and Cancer study $(\mathrm{N}=382)$ as a validation dataset. For each of the participant, genome wide methylation profiles were acquired from blood samples collected at enrolment using the Illumina HM450 DNA methylation array. We performed epigenome wide association studies within each dataset to assess the relation between methylation levels and smokingrelated variables, controlling for technical variation (batch effects) and confounding factors (including white blood cell composition). 
Results We found 8 and $897 \mathrm{CpG}$ sites differentially methylated in former and current smokers, while compared to never smokers, respectively. The 8 candidate markers of former smoking showed a gradual reversion of their methylation levels from those typical of current smokers to those of never smokers. Further analyses using cumulative (over varying time windows) smoking intensities, highlighted three classes of biomarkers: short and long term biomarkers (measuring the effect of smoking in the past 10, and in the past 10 to 30 years respectively), and lifelong biomarkers detected more than 30 years after quitting smoking.

Conclusions Genome-wide DNA methylation profiles show promising abilities to detect short-term to lifelong biomarkers of tobacco smoke exposure and, more generally, to potentially identify time-varying biomarkers of exposure.

\section{NIGHT WORK AND BREAST CANCER RISK AMONG WOMEN IN THE PUBLIC DANISH HEALTH CARE SECTOR - A SHORT-TERM FOLLOW UP OF A LARGE SCALE POPULATION}

${ }^{1}$ Helene Tilma Vistisen, ${ }^{2}$ Anne Helene Garde, ${ }^{2,3}$ Aase Marie Hansen, ${ }^{4}$ Johnni Hansen,
${ }_{5,6}$ Peer Christiansen, ${ }^{1}$ Henrik Kolstad. ${ }^{1}$ Department of Occupational Medicine, Danish
Ramazzini Centre, Aarhus University Hospital, Aarhus, Denmark; ${ }^{2}$ National Research Center
for the Working Enviroment, Copenhagen, Denmark; ${ }^{3}$ Department of Public Health,
University of Copenhagen, Copenhagen, Denmark; ${ }^{4}$ Danish Cancer Society Research
Center, Copenhagen, Denmark; ${ }^{5}$ Department of Surgery, P, Aarhus University Hospital,
Aarhus, Denmark; ${ }^{6}$ Danish Breast Cancer Corporative Group, Copenhagen, Denmark

10.1136/oemed-2014-102362.93

Objectives Experimental evidence suggest a short-term effect of light at night on breast cancer oncogenesis. We studied the short-term effect of night work on breast cancer occurence.

Method We established a large, national cohort of employees in the public health care sector with a high prevalence of night shift work and with detailed data regarding occupational title and date and hour for beginning and end of every work duty: The Danish Working Hour Database (DWHD). DWHD encompasses payroll data as of 2007 and is updated on an annual basis. For this analysis we defined night work as at least $3 \mathrm{~h}$ of work between midnight and 05:00. From national cancer registers we retrieved information about breast cancer diagnosis for all female workers and their relatives. Reproductive history, hormone medications, attendance in mammography screening, and vital status were obtained from other national health registries.

Results The 6-year follow up from 2007 to 2012 included 169.011 women of which 98.297 (58\%) had ever worked nights during the follow up. A total of 1.281 breast cancer cases occurred within the study population. 846 cases occurred among women never worked nights and 435 cases among women ever worked nights.

Conclusions Internal risk assessment of this dataset that includes alternative exposure metrics based on day-to-day night work exposure information will be presented.

\section{MARGINAL STRUCTURAL MODELS IN OCCUPATIONAL EPIDEMIOLOGY: AN APPLICATION IN THE US ALUMINIUM INDUSTRY}

${ }^{1}$ Andreas Neophytou, 'Daniel Brown, ${ }^{1}$ Sadie Costello, ${ }^{1}$ Elizabeth Noth, ${ }^{1}$ Katharine Hammond, ${ }^{2}$ Mark Cullen, 'Ellen Eisen. 'Environmental Health Sciences, UC Berkeley School of Public Health, Berkeley, CA, USA; ${ }^{2}$ Department of Internal Medicine, Stanford University, Stanford, CA, USA

10.1136/oemed-2014-102362.94
Objectives To apply Marginal Structural Models (MSM) to address healthy workers survivor effect in a cohort study of active workers when time varying variables on health status and exposure are measured.

Method We used Cox MSMs and inverse probability weighting to assess the effect of $\mathrm{PM}_{2.5}$ exposure on incident ischaemic heart disease (IHD) in an active cohort of 11966 US aluminium workers. The outcome was assessed using medical claims data from 1998 to 2012. Quantitative exposure metrics of current exposure to $\mathrm{PM}_{2.5}$ were dichotomized using different cutoffs and effects were assessed separately for smelters and fabrication. Risk score based on insurance claims was available as a time varying health status variable.

Results Defining binary PM2.5 exposure by the 10th percentile cut-off, health status was affected by past exposure and predicted subsequent exposure in smelters, but not in fabrication. A Traditional cox model was appropriate for fabricators; the hazard ratio was $1.51(95 \%$ CI: $1.12-2.06)$ and was attenuated when considering higher exposure cutoffs. In smelters, Cox MSM Hazard Ratios for IHD comparing the effect of exposure in a population had everyone always been exposed to everyone always unexposed, using the $10^{\text {th }}$ percentile exposure cutoff was 1.83 (95\% CI: 1.14 - 2.94). Higher exposure cutoffs also resulted in attenuated effects.

Conclusions Marginal Structural Models can be used in active employment occupational cohorts to address time varying confounding. Results from the current study suggest that occupational exposure to $\mathrm{PM}_{2.5}$ in the aluminium industry increases the risk of IHD in both smelters and fabrication.

\section{DIRECT EXPOSURE TO METALWORKING FLUID AEROSOLS AND CHRONIC OBSTRUCTIVE PULMONARY DISEASE IN A COHORT OF U.S. AUTOMOTIVE INDUSTRY WORKERS}

${ }^{1}$ Stella Beckman, ${ }^{1}$ Sadie Costello, 'Sally Picciotto, 1,2John Balmes, ${ }^{1} S$ Katharine Hammond, ${ }^{1}$ Ellen Eisen. 'Environmental Health Science, School of Public Health, University of California, Berkeley, Berkeley, CA, USA; ${ }^{2}$ Department of Medicine, University of California, San Francisco, San Francisco, CA, USA

\subsection{6/oemed-2014-102362.95}

Objectives Exposure to metalworking fluid (MWF) causes respiratory outcomes such as asthma and chronic bronchitis, as well as symptoms including phlegm and wheezing. Chronic obstructive pulmonary disease (COPD) encompasses these outcomes, and so is a potential result of MWF exposure. Recent evidence based on g-estimation suggests that reducing exposure to MWF would substantially decrease years of life lost due to COPD. The objective of this analysis is to examine the exposure-response relationship between direct exposure to MWF and COPD mortality in a large occupational cohort.

Method Hazard ratios were estimated using Cox proportional hazards models for the association between cumulative exposure to the thoracic fraction $\left(\mathrm{PM}_{9.8}\right)$ of straight, synthetic, or soluble MWF and COPD mortality. Subjects directly exposed to each fluid type were compared to those who were never directly exposed (assembly workers).

Results Hazard ratios for exposure quartiles increased in a nonmonotonic fashion, with a maximum of 1.6 for straight, 1.4 for soluble, and 1.5 for synthetic, reflecting an increased risk of COPD for exposed subjects. However, none of the HRs were significant at the 95\% confidence level. Indirect adjustment for 